\title{
Pursuing Sustainability with Process Integration and Optimization
}

\author{
Santanu Bandyopadhyay ${ }^{1}$ Dominic C. Y. Foo ${ }^{2} \cdot$ Raymond R. Tan $^{3}$
}

Published online: 12 May 2017

(C) Springer Science+Business Media Singapore 2017

Public concern about sustainability issues has grown significantly since the late twentieth century. This trend has been mirrored by similar growth in scientific literature dedicated to sustainability, particularly through the development of specialized areas of research within established disciplines. For example, searching the Scopus database using 'sustainability' as keyword in the subject area of chemical engineering results in close to 5000 listed publications to date. Significantly, the statistics show a sustained growth trend, culminating in 569 papers in 2016, which is an order of magnitude higher than the corresponding figures from the turn of the century. Thus, we can see clear evidence on how sustainability dimensions have permeated into chemical engineering literature (and, presumably to some extent, industrial practice as well). Similar statistical trends can be seen in allied disciplines such as energy engineering, mechanical engineering, environmental engineering, etc.

Dominic C. Y. Foo

ominic.foo@nottingham.edu.my

Santanu Bandyopadhyay

santanub@iitb.ac.in

Raymond R. Tan

raymond.tan@dlsu.edu.ph

1 Department of Energy Science and Engineering, Indian Institute of Technology Bombay, Powai, Mumbai 400076, India

2 Department of Chemical and Environmental Engineering/Centre of Excellence for Green Technologies, The University of Nottingham, Malaysia Campus, Broga Road, 43500 Semenyih, Selangor, Malaysia

3 Chemical Engineering Department, De La Salle University, 2401 Taft Avenue, 0922 Manila, Philippines
There are well-understood links between system efficiency and sustainability, which means there is significant potential for engineering tools originally developed for efficiency improvements to be redirected towards sustainable development with special emphasis on environmental goals. For example, mathematical programming models have been used in chemical engineering research since the 1950s, while process integration methods have been used since the 1970s for heat recovery and energy conservation. In recent years, mathematical optimization tools along with principles of process integration are applied towards developing sustainable systems.

Some historical developments for process integration in the past four decades are worth mentioning. The technique that was initiated in the 1970s has entered into its mature stage in late 1980s, with the establishment of the first research centre at the University of Manchester Institute of Science and Technology (UMIST; now University of Manchester). Various international meetings and workshops on process integration were held in the 1990s e.g. International Process Integration Conference (Gothenburg, 1992; Copenhagen, 1999), Process Integration Workshop on International Cooperation (Berlin, 1993), IEA Process Integration Implementing Agreement (1999). The annual process integration conference (PRES - Conference on Process Integration, Modelling and Optimisation for Energy Saving and Pollution Reduction) was held since 1998, in Prague (even years) and rotates in different countries (odd years). Two other important events in the twenty-first century were the Jubilee Conference (Gothenburg, 2013) and the recent IEA Expert Workshop on greenhouse gas mitigation (Berlin, 2017). It is easily observed that most developments on process integration were meant for energy conservation in the earlier days, and are very much diverted into environmental sustainability in the past two decades. 
Process Integration and Optimization for Sustainability (PIOS) is a new international journal dedicated to the development, utilization, and applications of systematic methodologies for improving the efficiency and reducing the environmental impacts of industrial processes and thereby aims to achieve overall sustainability. PIOS will provide a vehicle for presenting advances as well as best practices in the domain of process integration and mathematical optimizations with special emphasis on sustainability and aims to disseminate knowledge and to provide a fresh perspective regarding the basic concepts, theory, techniques and industrial applicability. PIOS aims not only to highlight the current research and developments but also to project the need and direction for further research in this subject. It will also provide a unique venue for sharing multidisciplinary research amongst undergraduate and graduate students, researchers in academia and professional practice consultants, contractors and government agencies.

The need for an effective and dedicated platform to appraise sustainability issues through publication of scientific research and industrial case studies were originally felt during the PRES conference in 2008. After close to a decade of laying the groundwork, this maiden issue of PIOS is now being published with an initial set of five articles covering such topics as industrial symbiosis, material recovery, biomass utilization, energy efficiency and crisis operations. We envision this initial issue of PIOS to be the promising start of a journal that will establish itself as a venue for dissemination of important contributions in this field, and welcome submissions from the global scientific community in our joint pursuit of sustainability. 